\title{
Emergence of a Promiscuous Peroxidase Under Non-Equilibrium Conditions
}

\author{
Sumit Pal ${ }^{1}$, Antara Reja ${ }^{1}$, Subhajit Bal ${ }^{1}$, Baishakhi Tikader², Sandip Kar ${ }^{2}$ and Dibyendu Das ${ }^{1 *}$
}

In the far from equilibrium state of living matter, energy is consumed to fuel the metabolic networks of enzymatic reactions. The emergence of protometabolic pathways in primeval earth is intricately related to the evolutionary journey of modern enzymes. Fundamental understanding of such energy driven generation of early catalytic systems would help us recognize the conditions required for the minimal metabolism that predated the chemical emergence of life. Herein, we report substrate driven generation of a non-equilibrium catalytic machinery from a single amino acid functionalized fatty acid in presence of a cofactor hemin. The non-equilibrium assembled state showed acceleration of catalytic potential resulting in degradation of the substrate and subsequently led to disassembly. Controls that promoted equilibration could not access the three-dimensional microphases and showed substantially lower catalytic activity. Significantly, the assembled state showed latent catalytic function by hydrolysing a precursor to yield the same substrate. Consequently, the assembly was benefitted with augmented lifetime of the catalytic state exploiting a promiscuous cascade and thus foreshadowing protometabolism. The results contribute towards our understanding of energy driven generation of primitive catalytic machineries that assisted the minimal metabolism of early life.

\footnotetext{
${ }^{1}$ Department of Chemical Sciences and Centre for Advanced Functional Materials, Indian Institute of Science Education and Research (IISER) Kolkata, Mohanpur 741246, India. Email: dasd@iiserkol.ac.in ${ }^{2}$ Department of Chemistry, Indian Institute of Technology Bombay, Powai, Mumbai 400076, India.
} 
Living organisms exist seamlessly at an entropically forbidden state that is sustained by a continuous expense of energy following the principles of non-equilibrium thermodynamics. ${ }^{1-6}$ Cell dynamics, functions and homeostasis are maintained under such non-equilibrium conditions by exploiting chemical energy stored in the thermodynamically activated molecules via metabolic networks of enzymatic reactions. ${ }^{7-12}$ Extant enzymes and cytoskeleton proteins manipulate energy consumption pathways as an evolutionary strategy across life forms to drive the functional microphases and carry out work with remarkable spatiotemporal control. ${ }^{7-10}$ The evolutionary journey of modern enzyme is intricately related to the emergence of metabolic pathways. ${ }^{10,13-17}$ Deep phylogenetic analyses have suggested the presence of highly conserved enzymes for amino acid and lipid metabolism. ${ }^{10}$ Minimal metabolic cycles involved with breaking of activated molecules and accessing of self-aggregated complex structures operating away from equilibrium, could in principle have set the stage for selection and adaptation. ${ }^{1,4}$ Different models have suggested theories about evolution of metabolic pathways, from recruitment of catalyst for creating depleted substrates to promiscuous activity. ${ }^{10}$ From the context of chemical origin of life working far from equilibrium, it is tempting to investigate mixtures of simple molecules under a flux of energy and subsequently probe the emergence of primitive catalytic machineries with latent functions that could be important for early metabolism. ${ }^{18-30}$ Herein, we show substrate driven generation of an artificial peroxidase via the dissipative self-assembly of a minimal amphiphile in presence of a cofactor (hemin) in an oxidizing environment (Fig. 1). The ordered non-equilibrium supramolecular assemblies feature kinetic asymmetry in the energy consumption pathway as a time delayed acceleration of catalytic potential was observed upon assembly that resulted in deactivation from higher energy state (Fig. 1b). ${ }^{1,2,6,23-26}$ Significantly, the higher energy nonequilibrium state showed promiscuous catalytic function to generate its own substrate featuring a two-step cascade reaction.

\section{Results and discussion}

We started with a combination of a simple amphiphile and a low molecular weight co-factor under an oxidizing environment (Fig. 1). For amphiphile, single amino acid (L-histidine) installed with a stearic acid was used (stearoyl histidine, SH, Fig. 1c). Hemin, an aromatic prosthetic group seen in modern peroxidases and metalloenzymes was used as a cofactor (Fig. 1a showing horseradish peroxidase, HRP as a representative example). The iron containing porphyrin moiety is featured at the active site of most heme-proteins and plays critical roles in energetically downhill oxidative processes that is important for metabolism. ${ }^{31,32} \mathbf{S H}(12 \mathrm{mM})$ alone or when mixed with hemin $(0.15 \mathrm{mM})$ remained as a free-flowing solution $\left(30 \% \mathrm{v} / \mathrm{v}, \mathrm{DMF} / \mathrm{H}_{2} \mathrm{O}, 22 \mathrm{mM} \mathrm{H}_{2} \mathrm{O}_{2}\right)$. We were curious whether the addition of a substrate could drive the non-equilibrium self-assembly of hemin and $\mathbf{S H}$. We further argued that the incorporation of a low molecular weight co-factor hemin may facilitate the energy dissipation via oxidative degradation of the electron rich substrate, resulting in return to equilibrium. Hence, the selection of the substrate was based on two criteria, firstly, it should be a substrate for extant peroxidase enzymes and secondly, it should be aromatic as planar moieties are known to induce assembly of fatty acid based amphiphiles. ${ }^{33,34}$ Based on these, planar aromatic molecule 4-chloro-1naphthol (CN) was selected (Fig. 1). ${ }^{34}$ Indeed, addition of $\mathrm{CN}(14 \mathrm{mM})$ resulted in the rapid selfassembly of $\mathbf{S H}(12 \mathrm{mM})$ in presence of hemin $(0.15 \mathrm{mM})$ as within ca. 30 min the solution turned into a self-supporting gel (Fig. 2). Notably, the color of the gel intensified gradually with time suggesting the onset of oxidation of the substrate in presence of hemin (Fig. 2d-f). Interestingly, after ca. $150 \mathrm{~min}$, the gel started to show an autonomous transition to sol state (Fig. 2f). It is important to note that in the absence of folded peptide chains containing proximally located histidine residues, native hemin has very low intrinsic peroxidase activity (Fig. 1a). ${ }^{32}$ However, the rapid color change observed in this case indicated a possible activation of hemin. 


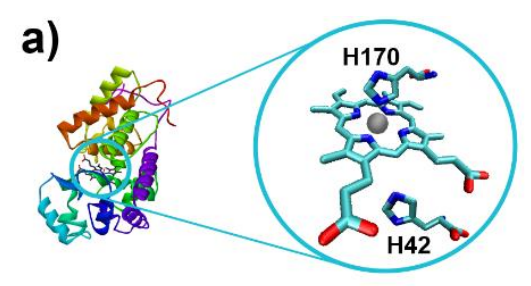

b)
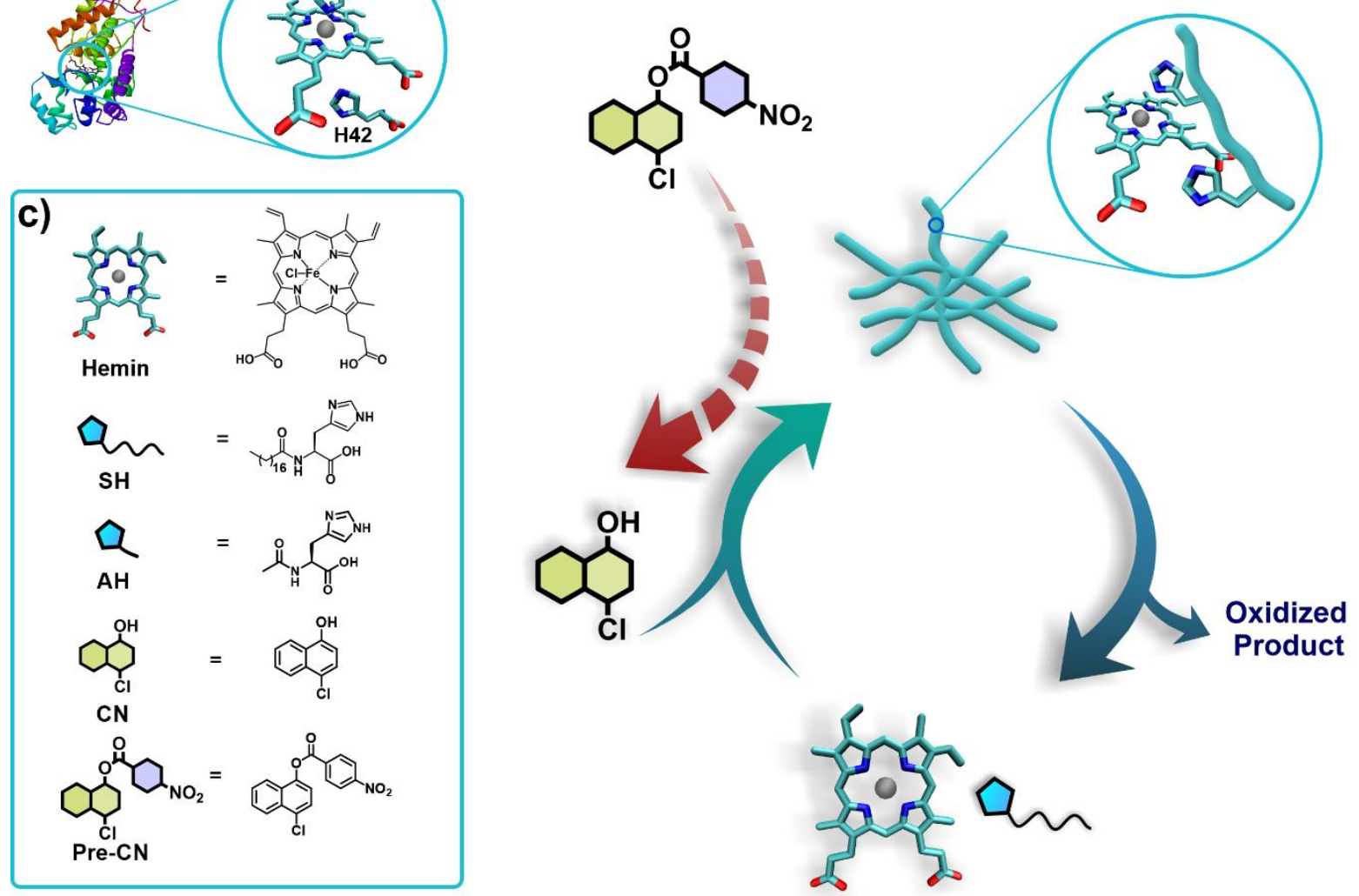

Fig. 1 I a) HRP displaying a cofactor-binding site. b) Schematic diagram representing the nonequilibrium dissipative system mimicking the peroxidase activity. The substrate $\mathbf{C N}$ drives the nonequilibrium generation of catalytic microphases which subsequently degrades $\mathbf{C N}$. Enlarged version shows the interaction of the histidines of the nanofibers with co-factor hemin. The precursor ester of CN (Pre-CN) could be hydrolyzed to prolong the lifetime of microphases. c) Chemical structures of the involved molecules.

To check the morphological transition of the non-equilibrium system, extensive transmission electron microscopy (TEM, Fig. 2a-c) and scanning electron microscopy (SEM, Supplementary Fig. 1) were performed. Time resolved electron microscopy micrographs at different intervals ca. $2 \mathrm{~min}$, ca. $30 \mathrm{~min}$ and finally at ca. $150 \mathrm{~min}$, showed temporal accessing of a nanofibrillar structures. The number of fibrillar structures seen at ca. 2 min, gradually intensified with aging and accessed three-dimensional network like microphases at ca. $30 \mathrm{~min}$ (Fig. 2a,b). The TEM micrographs of the sol state (at ca. 150 min) showed a substantial decrease of fibrillar networks, thus suggesting the temporal generation of nanofibrillar microphases (Fig. 2c). Further, SEM demonstrated similar temporal generation of network like morphologies (Supplementary Fig. 1). Next, fluorescence spectroscopy was executed to gain insights into the autonomous phase transition. Nile Red was used as a fluorophore $\left(50 \mu \mathrm{M}, \lambda_{\mathrm{ex}}=\right.$ $550 \mathrm{~nm}$ and $\lambda_{\mathrm{em}}=650 \mathrm{~nm}$ ) to monitor the microenvironment of the non-equilibrium self-assembled state. Nile Red is known to report the hydrophobic environment of self-assembled networks by showing increase in fluorescence intensity due to inhibition of the non-radiative decay pathways. The addition of Nile Red from the beginning showed a gradual increase of the fluorescence intensity till ca. $30 \mathrm{~min}$ (Fig. 2j). Expectedly, generation of hydrophobic environment was suggested from the initial increase of fluorescence intensity. After $30 \mathrm{~min}$, the intensity began to decrease over the next 150 min, suggesting the autonomous process of disassembly. Further, to visualize the autonomous transition in solution phase, Nile Red stained microphases were imaged at different time intervals with confocal laser scanning microscopy (CLSM, Fig. 2g-i). Temporal generation of fluorescent 

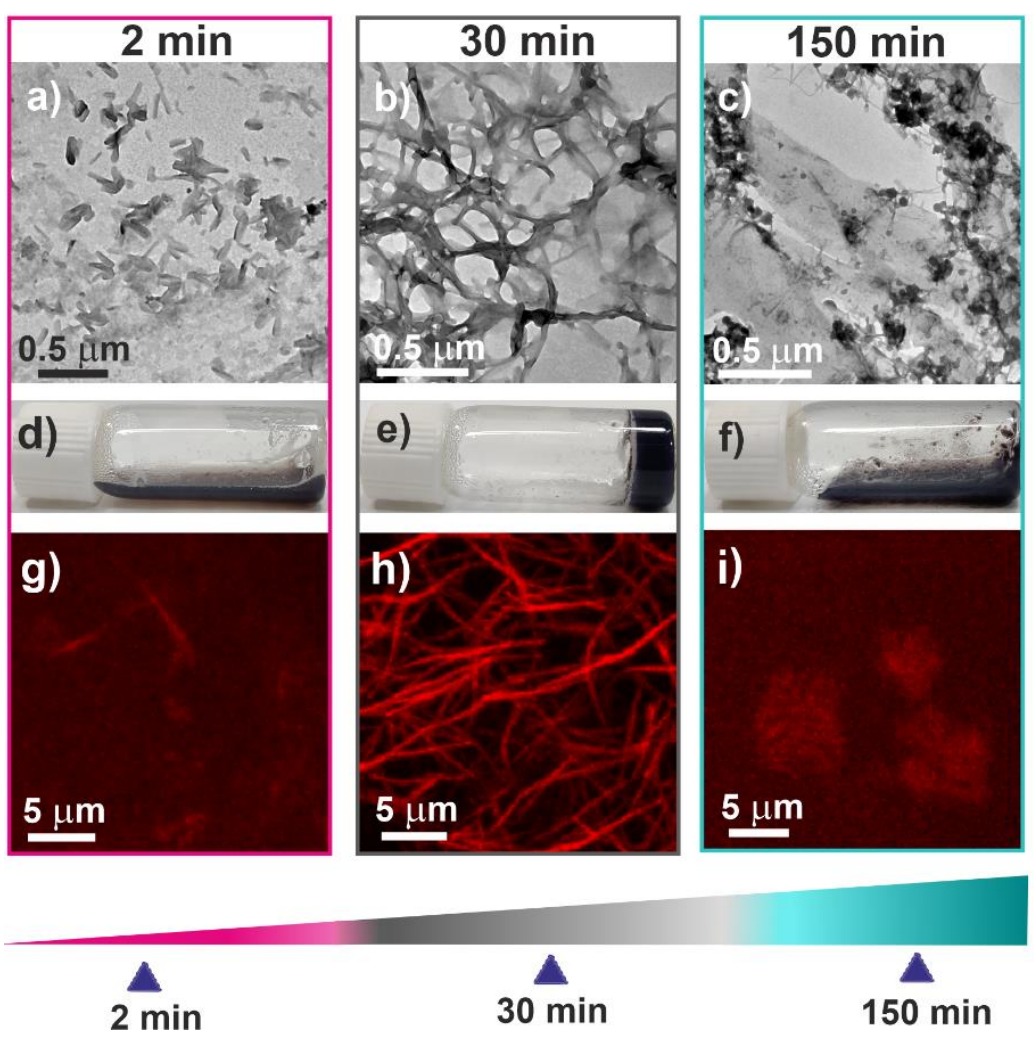

Fig. 2 I Time resolved TEM images of nonequilibrium dissipative system at a) 2 min (sol), b) 30 min (gel), c) $150 \mathrm{~min}$ (sol). Vial images of d) sol, e) gel and f) sol, show the phase change of the nonequilibrium dissipative system with time. CLSM images of $\mathbf{g}$ ) sol, $\mathbf{h}$ ) gel, i) sol. Temporal change in $\mathbf{j}$ ) fluorescence intensity $\mathbf{k}$ ) Change in mechanical property of the non-equilibrium dissipative system with time. Condition: SH (12 mM), $\mathrm{H}_{2} \mathrm{O}_{2}(22 \mathrm{mM})$, Hemin $(0.15 \mathrm{mM})$ and $\mathrm{CN}(14 \mathrm{mM})$. Error bars represent standard deviations of triplicate.

fibrillar microphases were observed with time (Fig. $2 \mathrm{~g}$-i). Rheological measurements were performed to investigate the mechanical properties of the non-equilibrium microphases. Time dependent rheology revealed the augmentation of storage modulus ( $\left.G^{\prime}\right)$ with time and registered the highest $G^{\prime}$ value at around $40 \mathrm{~min}$ followed by rapid decline with time, suggesting autonomous loss of mechanical strength (Fig. 2k).

What surprised us was the rapid formation of colored oxidized product due to degradation of the $\mathbf{C N}$ by the unfunctionalized hemin which is known to have low intrinsic peroxidase like activity. To probe the interaction of hemin with $\mathbf{S H}$ in the assembled state, UV/Vis spectroscopy was performed to monitor the binding of hemin (Fig. 3a). Free hemin had the Soret peak at $399 \mathrm{~nm}$ along with a shoulder at around $360 \mathrm{~nm} .{ }^{32}$ Further, a low intensity Q-band around $640 \mathrm{~nm}$ indicated the presence of $\mu$-oxo bihemin. However, hemin in presence of SH under identical conditions showed a significant red shift from $399 \mathrm{~nm}$ to $406 \mathrm{~nm}$ and a weak band at $570 \mathrm{~nm}$ (Fig. 3a). These changes in UV/Vis spectrum of hemin in presence of $\mathbf{S H}$ clearly suggested the interaction of hemin with histidine. To further probe the enhancement of activity, rate of oxidation was followed spectrophotometrically by monitoring the appearance of peak at $550 \mathrm{~nm}$ (Fig. 3c, Supplementary Fig. 2, see SI for details). ${ }^{34}$ Indeed, the nonequilibrium microphases of $\mathbf{S H}$ showed rapid peroxidase activity with $v_{i}=2.35 \pm 0.52 \mu \mathrm{M} / \mathrm{min}$. Further, the consumption of $\mathbf{C N}$ with time was monitored via HPLC chromatograms (Fig. $3 \mathrm{~d}$ ). To probe that the higher catalytic activity was generated from the temporally formed amphiphilic microphases, we used a control system which lacked the C18 chain keeping other parameters identical. 
a)

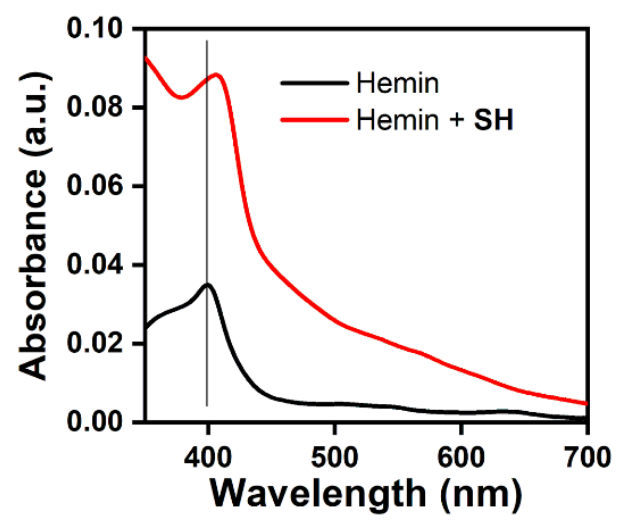

b)

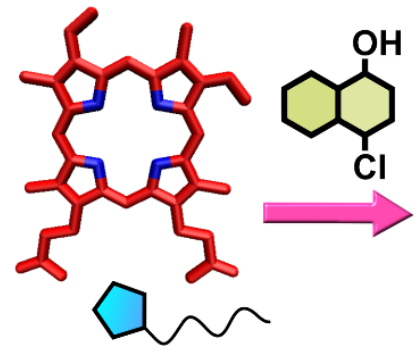

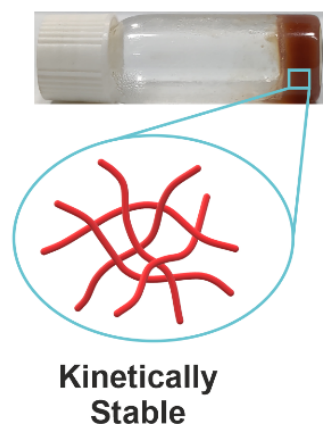

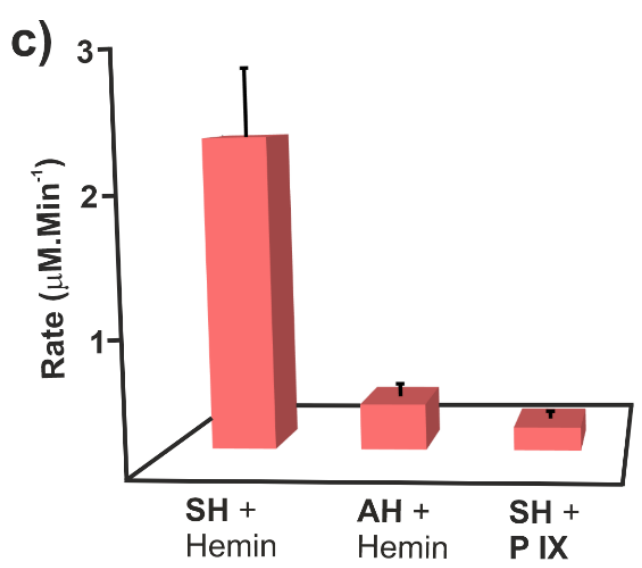

d)

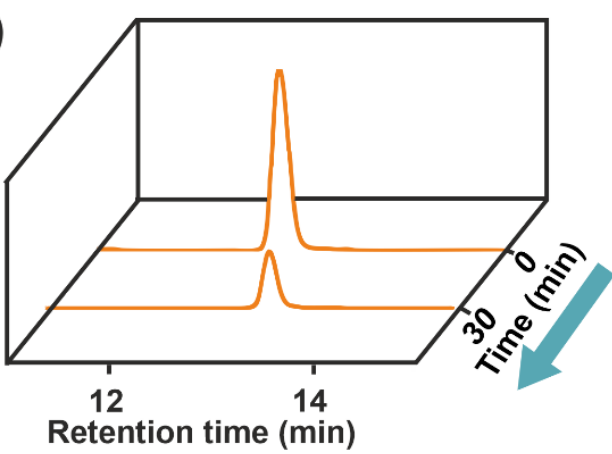

Fig. $3 \mathrm{I}$ a) Change in absorbance of hemin $(0.15 \mathrm{mM})$ Soret band in presence of SH (12 mM). b) Schematic diagram of the kinetically trapped gel formation in presence of $\mathbf{P}$ IX $(0.15 \mathrm{mM})$ instead of using hemin keeping all other components same. c) Bar diagram showing initial peroxidase rate of system (SH + hemin), control system (AH + hemin) containing C2 lipidated chain instead of using C18 chain and kinetically trapped system (SH + P IX). d) Time dependent HPLC displaying the consumption of $\mathrm{CN}$. Error bars represent standard deviations of triplicate.

For this purpose, histidine was installed with an acetyl $(\mathbf{C} 2, \mathbf{A H})$ group instead of a $\mathrm{C} 18$ chain and expectedly, $\mathbf{C N}$ was unable to drive $\mathbf{A H}$ to access network like microphases as seen for $\mathbf{S H}$ (representative TEM image in Supplementary Fig. 3). Significantly, AH showed a decline of rate of almost 7-fold of magnitude $\left(v_{i}=0.34 \pm 0.09 \mu \mathrm{M} / \mathrm{min}\right.$, Fig. 3c, Supplementary Fig. 4). This suggested that the dissipative generation of catalytic microenvironment in case of $\mathbf{S H}$ was responsible for the higher catalytic performance (Figs $1 \mathrm{~b}$ and $4 \mathrm{a}$ ). Further, we were curious to check whether a nondissipative kinetically trapped state can be accessed if we use catalytic defunct congener of hemin. For this purpose, we used protoporphyrin IX (P IX) which lacked the metal center ( $\mathrm{Fe}$ ) and thus would lead to the generation of a protoapoenzyme. Indeed, we observed the formation of gel which was kinetically stable for prolonged time (Fig. 3b). This control experiment underpins the importance of catalytic degradation of the substrate to dissipate energy aiding the return path to equilibrium. To investigate the reason for the significant enhancement of catalytic performance and generation of peroxidase mimic under non-equilibrium conditions (Fig. 4a), the order of addition was altered to preferentially direct the formation of equilibrium (non-dissipative) assemblies (Fig. 4b). For this purpose, SH was incubated along with hemin in the absence of $\mathbf{C N}$ under identical conditions as used before. After $12 \mathrm{~h}$ of incubation, $\mathbf{C N}$ was added to the system (Fig. 4b). We expected, hemin and $\mathbf{S H}$ will equilibrate to access non-dissipative self-assembled structures. Due to the time delayed addition, the substrate $\mathbf{C N}$ will be incapable to drive the non-equilibrium generation of the dissipative fibrillar networks with catalytic potential. Indeed, $\mathrm{CN}$ was unable to drive the formation of self-supporting gel when it was added to the premixed solution of $\mathbf{S H}$ and hemin in oxidizing environment (Fig. $4 \mathrm{~b}$ ). 
a) Non-equilibrium (dissipative) system
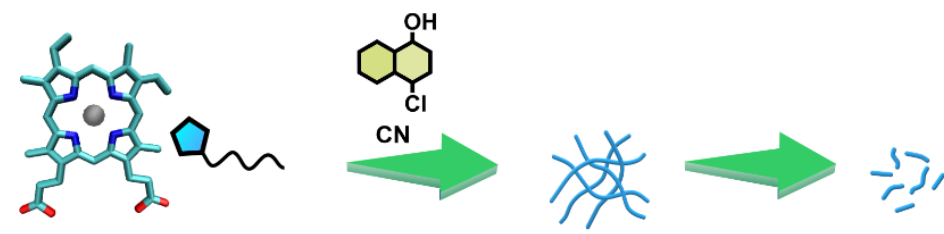

- Gel

b) Equilibrium (non-dissipative) system
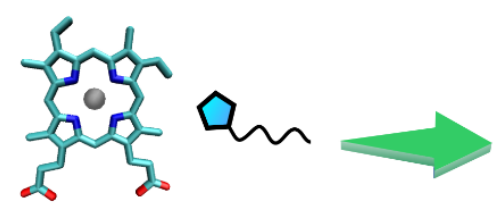

c) Hydrolysis

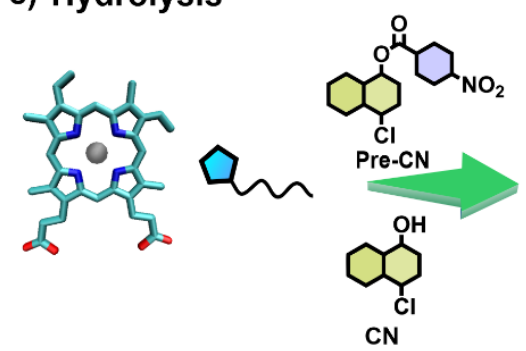

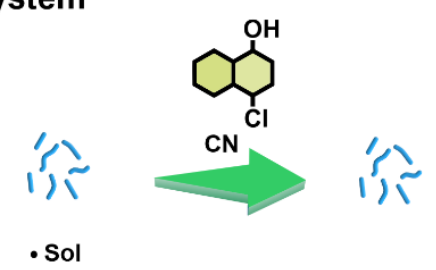

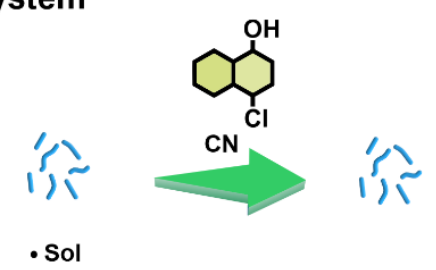

- Sol

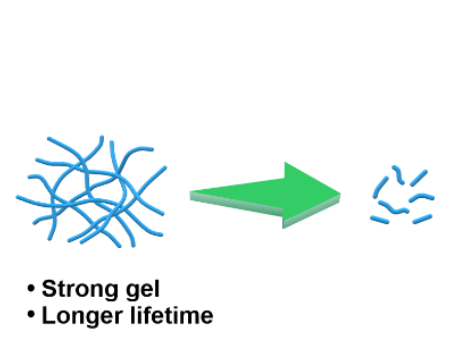

d)

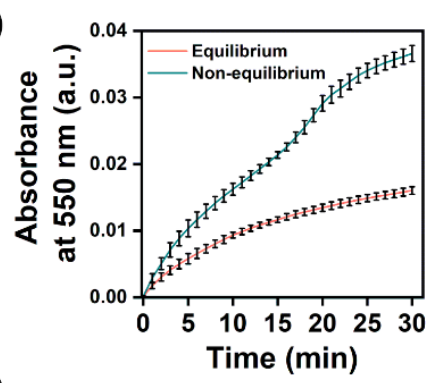

e)

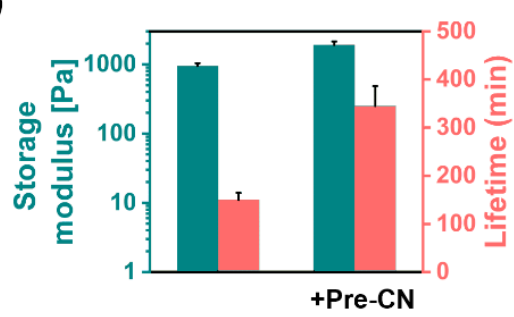

f)

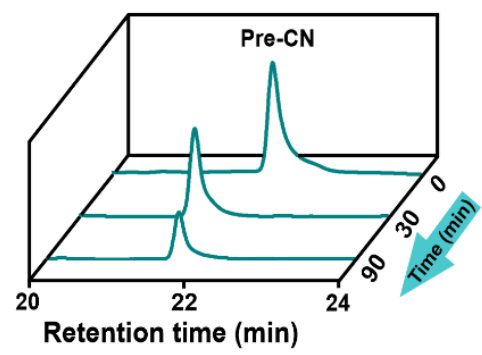

Fig. 4 I Schematic diagram of a) non-equilibrium dissipative system, b) equilibrium non-dissipative system and c) non-equilibrium dissipative system containing Pre-CN. d) Change in absorbance of $\mathbf{C N}$ at $550 \mathrm{~nm}$ of the systems. e) Change in storage modulus and the lifetime of the gel in presence and absence of pre-CN. f) HPLC chromatogram showing the consumption of Pre-CN with time. Error bars represent standard deviations of triplicate.

Extensive TEM investigations demonstrated the presence of only short fibrils at different time intervals without any networked morphologies for the equilibrated samples both in presence and absence of CN (Supplementary Fig. 5). Rheological studies confirmed lack of gel like behavior in the non-dissipative system (Supplementary Fig. 6). Further, the storage modulus did not show any temporal change of the viscoelastic property (Supplementary Fig. 6). Hence, the microscopic and rheological studies suggested that the substrate $\mathbf{C N}$ was unable to drive the formation of fibrillar network like morphology under the equilibrium promoting conditions. Finally, we monitored the rate of the oxidation for the equilibrated non-dissipative system. Kinetic measurement done at $550 \mathrm{~nm}$ showed a rate of $v_{i}=1.34 \pm 0.32 \mu \mathrm{M} / \mathrm{min}$. Importantly, the initial rate for this equilibrated mixture was found to be $\sim 60 \%$ of the non-equilibrium dissipative system (Fig. $4 \mathrm{~d}$ ). It is important to note here, it has been well established that supramolecular systems working under equilibrium conditions show improved catalytic activities compared to their corresponding building blocks. ${ }^{35-40}$ However, the data presented in this work underpins the fact that when a system is driven away from equilibrium, emergent catalytic microphases can be accessed which are even more proficient than the systems under equilibrium. ${ }^{25}$

Biological systems embody metabolic networks featuring numerous reaction cascades that are fueled by thermodynamically activated esters such as ATP. The evolutionary appearance of enzymes was preceded by such chemical networks that eventually formed the backbone of existing metabolic cycles. We were curious to investigate whether an activated precursor molecule can be metabolized by the present system for generating its own substrate and thus benefitting in terms of improved lifetime and robustness of the emergent catalytic structures. For this purpose, the $\mathbf{C N}$ was esterified 
to access the thermodynamically activated ester Pre-CN (Figs 1 and 4c). It was argued that the Pre-CN ester can be hydrolyzed to $\mathbf{C N}$ by the non-equilibrium structures of $\mathbf{S H}$ featuring an arrangement of solvent exposed histidines. The released $\mathbf{C N}$ will subsequently be oxidized and the system will demonstrate catalytic promiscuity with a hydrolase-peroxidase cascade. Indeed, Pre-CN (2 mM) was rapidly consumed and subsequently generated $\mathrm{CN}$ as followed from HPLC with a rate of $12.7 \mu \mathrm{M} / \mathrm{min}$ (Fig. 4f, SI for details). Notably, the lifetimes of the gels increased significantly from ca. $2 \mathrm{~h}$ to $5 \mathrm{~h}$ on triplicate measurements (Fig. 4e). Further, mechanical strength measurements by rheological studies supported significant improvement of storage modulus values (Fig. 4e and Supplementary Figs 7-10). These results underpinned the emergent promiscuity and the protometabolic role of the hydrolaseperoxidase cascade was facilitating the fitness of the catalytic higher energy states; the hydrolytic step helping in the generation of the substrate while the oxidation step facilitating the energy dissipation via oxidation.

\section{Conclusions}

Far from equilibrium life is sustained by the continuous expense and dissipation of energy via complex metabolic networks featuring numerous fuel driven catalytic conversions. Fundamental understanding about the evolutionary journey of extant enzymes would help us recognize primitive catalytic machineries and conditions of minimal metabolism predating the chemical emergence of life. Herein, we have shown a single amino acid-based lipid which in presence of a cofactor hemin can be driven by simple substrates to access non-equilibrium catalytic self-assemblies. The driven dissipative state shows emergence of peroxidase activity with enhanced catalytic performance under nonequilibrium conditions; thus, featuring a kinetic asymmetry in the energy consumption pathway. When a control porphyrin without Fe as a catalytically defunct co-factor was used, a kinetically trapped assembled state was accessed. This state had significantly lesser oxidation rates and hence was incapable to dissipative energy which led to prolonged kinetic stability. Moreover, control systems that promoted equilibration, the three dimensional catalytic microenvironment was never accessed and hence had significantly lower catalytic activity. Notably, the present system of simple building blocks showed crucial advantage in terms of emergence of catalytic promiscuity for facilitating two step cascade transformations. The hydrolytic role added as a secondary loop was beneficial for the overall catalysis, as this latent role helped in augmenting the lifetime of the catalyst for the oxidation reaction. The non-linearity in the different catalytic conversions' benefits the emergence of a particular chemical transformation.

The dissipative formation of catalytic peroxidases and the demonstration of the promiscuous cascade activity provides a functional feedback and sets the stage for the onset of protometabolism with an opportunity for cooption. The away from equilibrium catalytic microphases generated from simple precursors have potential for stochastic innovation as a bridge between biotic and abiotic world and can offer a platform for developing a general theory of chemical evolution that predates an emerging metabolism..$^{5,41-45}$ Systems chemistry approach such as these will eventually help us to define the physical constraints on the argument of chemical emergence of metabolic life. 


\section{References}

1. Ragazzon, G. \& Prins, L. J. Energy consumption in chemical fuel-driven self-assembly. Nat. Nanotechnol. 13, 882-889 (2018).

2. Astumian, R. D. Kinetic asymmetry allows macromolecular catalysts to drive an information ratchet. Nat. Commun. 10, 3837 (2019).

3. Whitesides, G. M. \& Grzybowski, B. Self-assembly at all scales. Science 295, 2418-2421 (2002).

4. England, J. Dissipative adaptation in driven self-assembly. Nat. Nanotechnol. 10, 919-923 (2015).

5. Astumian, R. D. Stochastic pumping of non-equilibrium steady-states: how molecules adapt to a fluctuating environment. Chem. Commun. 54, 427-444 (2018).

6. Penocchio, E., Rao, R. \& Esposito, M. Thermodynamic efficiency in dissipative chemistry. Nat. Commun. 10, 3865 (2019).

7. Hess, H. \& Ross, J. L. Non-equilibrium assembly of microtubules: from molecules to autonomous chemical robots. Chem. Soc. Rev. 46, 5570-5587 (2017).

8. David-Pfeuty, T., Erickson, H. P. \& Pantaloni, D. Guanosinetriphosphatase activity of tubulin associated with microtubule assembly. Proc. Natl Acad. Sci. USA 74, 5372-5376 (1977).

9. Caplow, M. \& Shanks, J. Mechanism of the microtubule GTPase reaction. J. Biol. Chem. 265, 8935-8941 (1990).

10. Carbonell, P., Lecointre, G. \& Faulon, J. L. Origins of specificity and promiscuity in metabolic networks. J. Biol. Chem. 286, 43994-44004 (2011).

11. Leveson-Gower, R. B., Mayer, C. \& Roelfes, G. The importance of catalytic promiscuity for enzyme design and evolution. Nat. Rev. Chem. 3, 687-705 (2019).

12. Semenov, S. Y. et al. Rational design of functional and tunable oscillating enzymatic networks. Nat. Chem. 7, 160-165 (2015).

13. Pappas, C. G. et al. Emergence of low-symmetry foldamers from single monomers. Nat. Chem. 12, 1180-1186 (2020).

14. Greenwald, J., Friedmann, M. P. \& Riek, R. Amyloid aggregates arise from amino acid condensations under prebiotic conditions. Angew. Chem. Int. Ed. 55, 11609-11613 (2016).

15. Carny, O. \& Gazit, E. A model for the role of short self-assembled peptides in the very early stages of the origin of life. FASEB J. 19, 1051-1055 (2005).

16. Tamura, T. \& Hamachi, I. Chemistry for covalent modification of endogenous/native proteins: from test tubes to complex biological systems. J. Am. Chem. Soc. 141, 2782-2799 (2019).

17. Gentile, K., Somasundar, A., Bhide, A. \& Sen, A. Chemically powered synthetic "living" systems. Chem 6, 2174-2185 (2020). 
18. Ashkenasy, G., Hermans, T. M., Otto, S. \& Taylor, A. F. Systems chemistry. Chem. Soc. Rev. 46, 2543-2554 (2017).

19. Azevedo, H. S., Perry, S. L., Korevaar, P. A. \& Das, D. Complexity emerges from chemistry. Nat. Chem. 12, 793-794 (2020).

20. Ottelé, J., Hussain, A.S., Mayer, C. \& Otto, S. Chance emergence of catalytic activity and promiscuity in a self-replicator. Nat. Catal. 3, 547-553 (2020).

21. te Brinke, E. et. al. Dissipative adaptation in driven self-assembly leading to self-dividing fibrils. Nat. Nanotechnol. 13, 849-855 (2018).

22. Tian, L. et al. Non-equilibrium spatiotemporal sensing within acoustically patterned twodimensional protocell arrays. ACS Cent. Sci. 4, 1551-1558 (2018).

23. Muñana, P. S. et al. Substrate-induced self-assembly of cooperative catalysts. Angew. Chem. Int. Ed. 57, 16469-16474 (2018).

24. Bal, S., Ghosh, C., Ghosh, T., Vijayaraghavan, R. K. \& Das, D. Non-equilibrium polymerization of cross- $\beta$ amyloid peptides for temporal control of electronic properties. Angew. Chem. Int. Ed. 59, 13506-13510 (2020).

25. Chen, R., Neri, S. \& Prins, L. J. Enhanced catalytic activity under non-equilibrium conditions. Nat. Nanotechnol. 15, 868-874 (2020).

26. Afrose, S, P., Bal, S., Chatterjee, A., Das, K. \& Das, D. Designed negative feedback from transiently formed catalytic nanostructures. Angew. Chem. Int. Ed. 58, 15783-15787 (2019).

27. Deng, J. \& Walther, A. ATP-powered molecular recognition to engineer transient multivalency and self-sorting 4D hierarchical systems. Nat. Commun. 11, 3658 (2020).

28. Boekhoven, J. et al. Transient assembly of active materials fuelled by a chemical reaction. Science 349, 1075-1079 (2015).

29. Debnath, S., Roy, S. \& Ulijn, R. V. Peptide nanofibers with dynamic instability through nonequilibrium biocatalytic assembly. J. Am. Chem. Soc. 135, 16789-92 (2013).

30. Sawczyk, M. \& Klajn, R. Out-of-equilibrium aggregates and coatings during seeded growth of metallic nanoparticles. J. Am. Chem. Soc. 139, 17973-17978 (2017).

31. Chatterjee, A., Mahato, C. \& Das, D. Complex cascade reaction networks via cross $\beta$ amyloid nanotubes. Angew. Chem. Int. Ed. 60, 202-207 (2021).

32. Wang, Q. et. al. A supramolecular-hydrogel-encapsulated hemin as an artificial enzyme to mimic peroxidase. Angew. Chem. Int. Ed. 46, 4285-4289 (2007).

33. Lee, H. Y., Nam, S. R. \& Hong, J.-I. Microtubule formation using two-component gel system. J. Am. Chem. Soc. 129, 1040-1041 (2007).

34. Ulyashova, M. M., Rubtsova, M. Y. \& Egorov, A. M. Colorimetric detection of immobilized horseradish peroxidase based on the co-oxidation of benzidine derivatives and 4-chloro-1naphthol. Russ. Chem. Bull. 60, 656-661 (2011).

35. Zhang, C. et. al. Switchable hydrolase based on reversible formation of supramolecular catalytic site using a self-assembling peptide. Angew. Chem. Int. Ed. 56, 14511-14515 (2017). 
36. Wei, G. et. al. Self-assembling peptide and protein amyloids: from structure to tailored function in nanotechnology. Chem. Soc. Rev. 46, 4661-4708 (2017).

37. Pengo, P., Polizzi, S., Pasquato, L. \& Scrimin, P. Carboxylateimidazole cooperativity in dipeptide-functionalized gold nanoparticles with esterase-like activity. J. Am. Chem. Soc. 127, 1616-1617 (2005).

38. Zhao, X. et al. Substrate-driven chemotactic assembly in an enzyme cascade. Nat. Chem. 10, 311-317 (2018).

39. Omosun, T. O. et. al. Catalytic diversity in self-propagating peptide assemblies. Nat. Chem. 9, 805-809 (2017).

40. Singh, N., Conte, M. P., Ulijn, R. V., Miravet, J. F. \& Escuder, B. Insight into the esterase like activity demonstrated by an imidazole appended self-assembling hydrogelator. Chem. Commun. 51, 13213-13216 (2015).

41. Epstein, I. R. Reaction: life is messy. Chem 5, 1917-1923 (2019).

42. Bai, Y. et al. Achieving biopolymer synergy in systems chemistry. Chem. Soc. Rev. 47, 54445456 (2018).

43. Xavier, J. C., Hordijk, W., Kauffman, S., Steel, M. \& Martin, W. F. Autocatalytic chemical networks at the origin of metabolism. Proceedings of the Royal Society of London. Series B: Biological Sciences 287, 20192377 (2020).

44. Deamer, D. \& Weber, A. L. Bioenergetics and life's origins. Cold Spring Harb. Perspect. Biol. 2, a004929 (2010).

45. Pross, A. How can a chemical system act purposefully? Bridging between life and non-life. J. Phys. Org. Chem. 21, 724-730 (2008). 


\section{Acknowledgment}

D.D. is thankful to SB/SJF/2020-21/08 Grant for financial assistance. S.P. and S.B. acknowledge CSIR India and A.R. acknowledges IISER Kolkata for fellowships.

\section{Author Contribution}

D.D. conceived and supervised the overall project. S.P, A.R, and S.B conceived and performed all the experiments. B.T and S.K conceived and carried out the kinetic modelling. D.D, S.P, A.R, and S.B cowrote the paper. All authors discussed the results and commented on the manuscript.

Corresponding Author

dasd@iiserkol.ac.in

ORCID Dibyendu Das:0000-0001-6597-8454

Table of content:

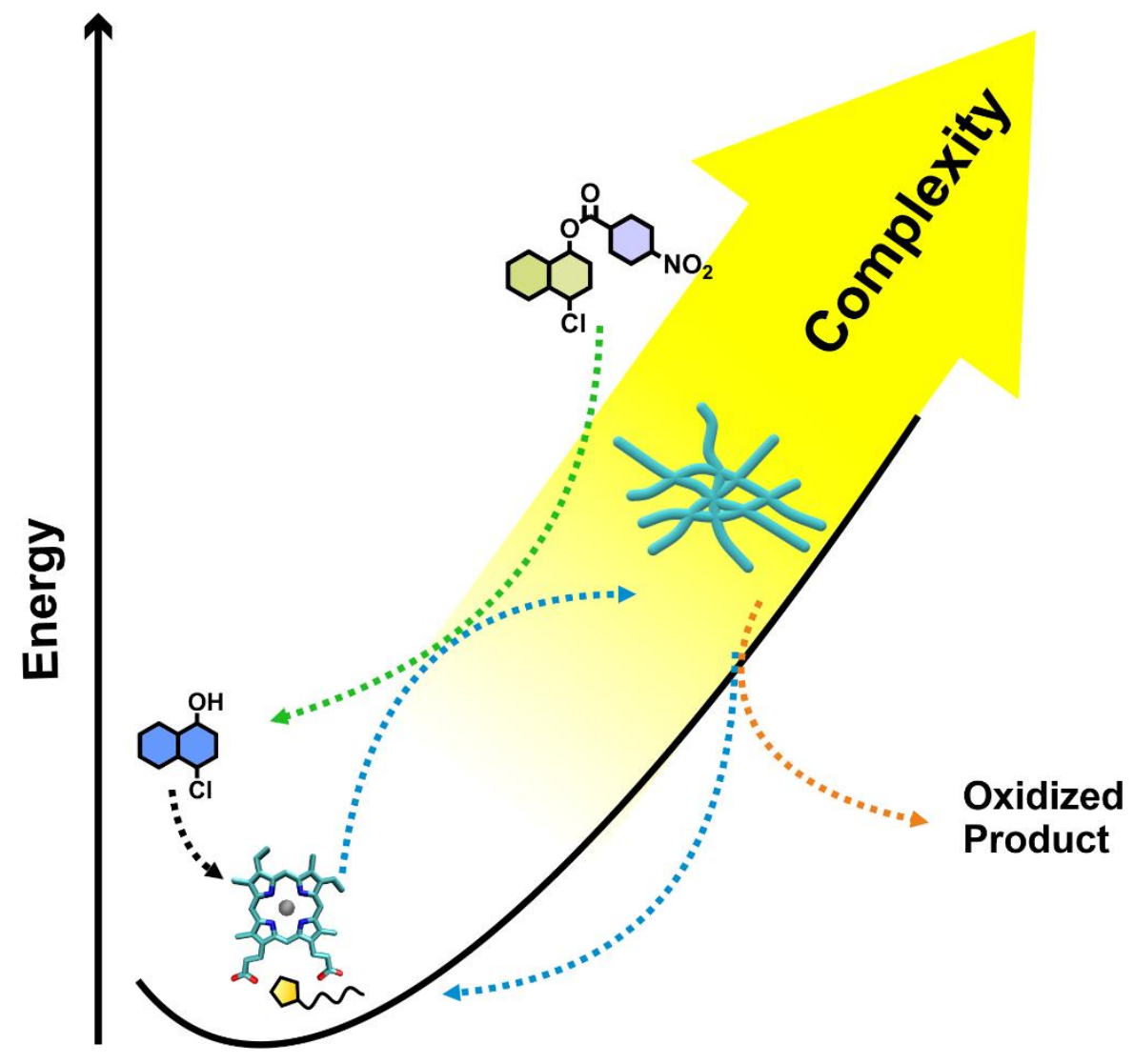

